\title{
Development of system for detecting hidden objects based on UWB pulse radar
}

\section{- Thang Tran-Dai}

- Tuan Do-Hong

- Ha Hoang-Manh

Ho Chi Minh city University of Technology, VNU-HCM, Vietnam

(Manuscript Received on July 15, 2015, Manuscript Revised August 30, 2015)

\begin{abstract}
This paper present a solution for detecting hidden objects based on UWB pulse radar. In this paper, we introduce an overview of UWB systems, the theory related and used in the process to implement the project. We present the steps to collect and

process data through object identification algorithm, improve algorithm for detecting hidden objects in some kind of environments. We evaluate the obtained results, conclude for applying orientation and development of the subject.
\end{abstract}

Keywords: UWB (Ultra Wideband) Radar, Migration algorithms, HS (Hyperbolic Summation).

\section{INTRODUCTION}

By using electromagnetic signals from Ultra Wideband (UWB) radiating in examined space and receiving electromagnetic signals at multiple points in space for analysis, wave propagation characteristics analysis system from the ultra wideband is capable of analyzing the characteristics of structure, materials and propagation of electromagnetic waves of the examined space with high spatial resolution, and wideband frequency spectrum. Therefore, applications as well as research implemented from this analysis system are very large, such as in: military, security, rescue, training, medical, construction, transportation, geology, $\operatorname{archeology} \ldots$

The limitation of technical and technological design, ultra-high frequency circuits design, ultra wideband antennas, high precision circuits, low noise, realizing signal processing algorithms linking from time to space are the major challenges for the system design. UWB systems have only been manufactured, used restrictedly in a few specific areas: military, security, research, geological... Vietnam is still limited in many problems that mentioned above, UWB analysis systems have not been made. Some applications for the purposes of geological surveying, quantity surveying, transportation construction defects, underground work, they have to use imported system, and these systems are only used for specific purposes with high cost rent or investment. And that is the reason for conducting 
this study to implement a wave propagation characteristics analysis system with ultra wideband applying for nondestructive structural analysis.

Although UWB systems have been popular for years but only recently really be noticeable in the wireless industry. UWB techniques are different from the wireless narrowband transmission techniques - replaced by transmissions on separate frequency channels, UWB signals spread over a wide frequency range. Typical forms of communication based on radio waves are replaced by sinusoidal pulse sequences with pulse rate of millions per second. With broadband and very small power, UWB signal is like background noise.

The field can be applied, deployed the results:

- Research and education: examination and nondestructive structural analysis, the study of material properties, propagation of electromagnetic waves, radio channels, characteristics of the transmission line type,...

- Medical: medical images, vital signs sensing: breathing, heart rate in distance,...

- Security, military: detect hidden objects, concealed weapons, buried landmines,...

- Construction: work quality checking (thickness, density...), determining status of cracking, pit, underground constructions...

- Salvage and rescue: detecting buried people, animals...

- Transportation: automatic driver support system, collision, obstruction avoiding...

- Reducing disaster damage: detection of pit, cleft in the river, dam, slopes caused by flooding, water level determining sensors...

- Archaeology, exploration: discovering of ancient monuments, buried bones, caves...

In this paper, we learn and carry out the installation, measure and examine objects using
UWB pulses radar. Then, we collect data for signal processing in the following steps. In the next step, we research advanced algorithm to detect objects and execute programs written in Matlab. Finally, we evaluate the system and algorithm through samples in different conditions.

\section{UWB SYSTEM}

\subsection{UWB Tranceiver}

The techniques which are often used in ultra wideband system: UWB pulse, frequency sweeping and spread spectrum. Each technology has its own characteristics and challenges for the design, fabrication. Based on the technical characteristics and the feasibility of the technique, UWB pulse technique was chosen because its characteristics can achieve high spatial resolution, high data acquisition speed and high feasibility.

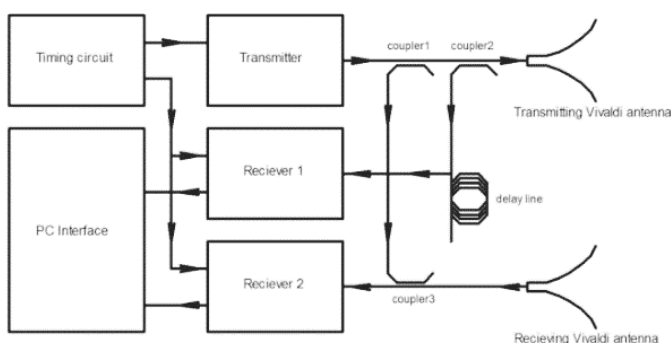

Figure 4. UWB Transceiver Diagram

The composition and functions of UWB transceiver:

- UWB transmitting circuit: transmitting signal cyclically.

- UWB receiving circuit 2: receiving signal from receiving antenna and a part of signal coupled directly from the UWB generator through coupler1 and coupler3. This signal acquisition is synchronized with UWB signals transmitted from UWB transmitting circuit. This receiver also assume the function of conversion Radio Frequency (RF) signal spectrum into Intermediate Frequency (IF) to be sampled by the Analog-toDigital Converter (ADC) with low speed in PC. 
- UWB receiver circuit 1 (receiver 1$)$ : receiving signal coupled directly from the output of UWB transmitting circuit through coupler2 UWB combining with delay line. This signal contains time information corresponding signal from UWB transmitting circuit and it is used for synchronization purposes and minimizing phase noise, effects of jitter. This signal acquisition is synchronized with UWB signals transmitted from UWB transmitting circuit. This receiver also take on the function of conversion RF signal spectrum into IF signal to be sampled by the low-speed ADC of PC.

- Timing circuit: generating signal with standard period for UWB generating and receiving circuit to generate the synchronization between the transmitting and receiving circuit as well as the standard offset for the frequency mixer.

- Coupler circuits and delay line: extracting, distributing signal and creating the standard delay time period for obtaining information signal synchronization between the transmitter and receiver, that is the basis to remove the jitter for the system.

- PC interface circuit: working as buffers for communicating with the PC data acquisition.

- UWB transceiver's features:

- Number of UWB transmitting channels: 01

- Number of UWB receiving channels: 02

- Frequency range: $1 \mathrm{GHz}-9 \mathrm{GHz}$

- Output power: low $(<-13 \mathrm{dBm})$

- Maximum Operating range: $~ 7.8 \mathrm{~m}$

- Spatial resolution/accuracy: $<1 \mathrm{~cm}$

- Dynamic range:> 40dB

- Signal refreshing rate: $\sim 40 \mathrm{~Hz}$

- Sampling cycle equivalent: $~ 21.7 p s$
UWB transceiver had been manufactured from spare parts and integrating into metal box size: $260 \times 210 \times 50 \mathrm{~mm}^{3}$.

\subsection{Antenna System}

The layout of transceiving antenna, UWB important influence to the operation of the system. Those are the factors that must be considered when integrating transmitting and receiving antennas into the system.

First, the transmission model of system from transmitting position to object and from the object to the receiving position may be in monostatic, bistatic, or multistatic. For achieving precise spatial coordinates to determine the location of transmitters and receivers point, as well as avoid the impact of the relative deviation of time during setup installation, two antennas for transmitting and receiving are placed and combined with the UWB transmitting and receiving signal into a fixed system. The distance between the transmitting and receiving antennas was selected accordingly to the system that can operate in either Pseudomonostatic or Bistatic mode.

Second, there is a direct signal coupling from transmitting antenna to the receiving antenna. Due to the distance between the transmitting and receiving antennas are much smaller than the distance from the transmitting/receiving antenna to the object, signal coupled directly to receiving antenna is very large. Meanwhile, the dynamic range of the UWB transmitter, receiver signals are limited. Thus to increase the sensitivity of the system, it should be minimized the direct coupling from the transmitting antenna to the receiving antenna.

Thirdly, it needs to obtain appropriate terms of polarization of the transmitting and receiving antenna with the highest probability for the scattering effects from the actual object.

The distance between the two ports transmit and receive UWB is chosen as $180 \mathrm{~mm}$. 


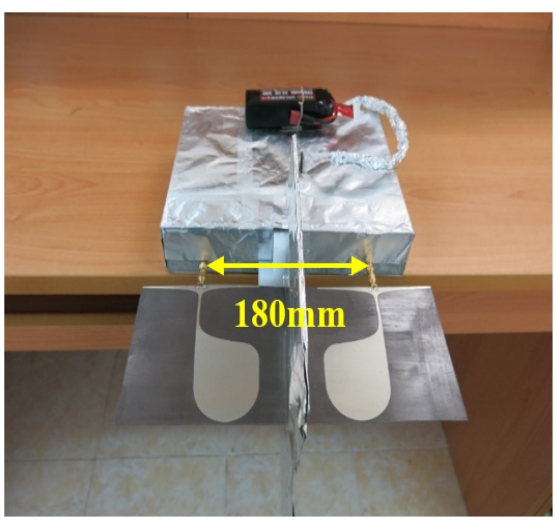

Figure 5. UWB Pulse Radar

\subsection{The Scanning and Space Positioning System}

Two overall structure of the system has been built. Two overall structure of the system are based on the scanning space. For the first system structure, electromagnetic radiation transceiving systems moving through in space in two dimensions $\mathrm{x}, \mathrm{y}$ thanks to the two-dimensional space scanner; object examined is fixed in space. For the second system architecture, electromagnetic radiation transceiving systems is fixed in space; object examined is rotated around an axis in space thanks to the rotating cylindrical system. This paper will focus on the use of the second system structure.

The change of signal after processing through the high-frequency circuit (IF signal) into a digital signal and the processing platform are necessary. The selection of analog converter - digital processing systems is based on the baseband signal bandwidth, signal-to-noise ratio achieved by the system and the speed of data acquisition. Besides, the elements of processing ability, processing system will also need to be considered: energy consumption, compatibility of communication between the processor system elements, platform for the development of algorithms. Collected composition and processed data obtained in this study is the PC, due to its significant features are easy to develop complex algorithms and features for data acquisition speed, bandwidth, signal to noise ratio, and its processing power can meet the needs of the system.

The collection of data is based on the ADC of PC's sound card. The requirements of data collection for this system is to collect 2 channels synchronously, 16 bits, sampling rate is 96 Ksample/s.

Signal processing, data processing of system can be classified into two main processes which are processes that improve quality of the received signal, and the application of the algorithm is based on the modeling of electromagnetic waves, the space-time relationship reflects on the signal transmitter/receiver to be able to get spatial structure information of space observation.

Due to the characteristics of the hardware system, in the process of implementing some of the system issues have been resolved:

- The PC's data acquisition is not synchronized with the signal from the UWB receiver and this signal has jitter (fluctuation, signal shift in small time ). The asynchronous and jitter affect the outcome after the reduction of environmental impact and its significant influence to the sensitivity of the system. This problem can be solved based on the re-sampling signal through interpolation processing. The process of resampling signal based on timing information of the reference signal at the start of each cycle. Information at the point of time in one cycle is necessary for re-sampling. Information at the point of time can be achieved based on the coupler2, delay line and receiver 1 receiver in the transmitter block diagram, UWB receivers.

- The signal from the UWB signal receiver is continuous, periodic. This signal needs to be split into separate exact cycle. The separation is done by generating characterizing signal at the start of each cycle. Coupling circuit 1 and 3 in the block diagram of the UWB transmitter, receiver undertake this function. Based on this characteristic, signal can be split into separate exact cycle. 


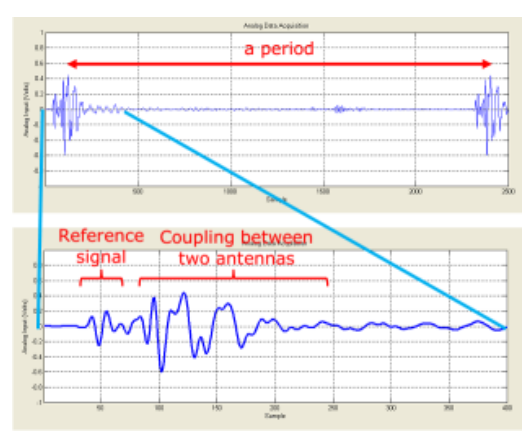

Figure 6. Continuously recirculating signal from the receiver UWB receiver and a reference feature can be added to separate into distinct exact period

- The mechanical vibrations in the scanning space for UWB transmitter and receiver signal also affect inclusion. Signal acquisition appears undesirable vibrations. The effects of mechanical vibration may be reduced based on the controls to stop the scanning space a sufficient time to turn off the mechanical oscillation before receiving signals from the UWB receiver combined with averaging multiple signal cycles over time.

Process data acquisition and signal processing:

- The space scanner is controlled to take UWB transceiver to determined location.

- Signals represent typical UWB electromagnetic waves were detected by the UWB transmitter, receiver at the specified location and were transmitted to the $\mathrm{PC}$.

- Continuous signal is decomposed into separate period based on the reference signal.

- At each stop position scanners, some cycles are collected and averaged to reduce the effects of mechanical vibration.

- The influence of the environment, direct signal coupling between the two antennas... is reduced based on environmental cues associated with medium spatial algorithms.

- The Hyperbolic summation algorithm (HS) and improved HS algorithm are used for spatial structure image reconstruction of examined space areas.

- Results are expressed as 2-dimensional image if only scanning one-dimensional space, or as a 3D image if using two-dimensional scanning.

Removing the influence of background:

- The influence of the environment, direct signal coupling between the two antennas... is reduced based on environmental cues associated with medium spatial algorithms.

- The signals of environmental cues were included in only the hardware components of the system, not with the measurable respondents. Sets these signals is used for reducing the influence of the direct signal between two antenna coupling, coupled through objects such as enclosure systems, cables, scanners and space navigation.

- The elimination of background influence also is associated with a medium spatial algorithm. This algorithm is similar to a spatial filter, takes the form of a high pass filter. Filter averages a set of continuous trace during the scan and eliminates it for each trace (horizontal filter). This filter is very effective in highlighting the weak signal invisible on the large background signal. However, this method can eliminate the important scattering signal components.

$$
g(x, y)=f(x, y)-\frac{1}{n} \sum_{i=-\frac{n}{2}}^{i=\frac{n}{2}} f(x, y+i)
$$

where $n$ is window size, $f(x, y)$ is the original data, $g(x, y)$ is image data which was removed the background.

Rotor and its components: 


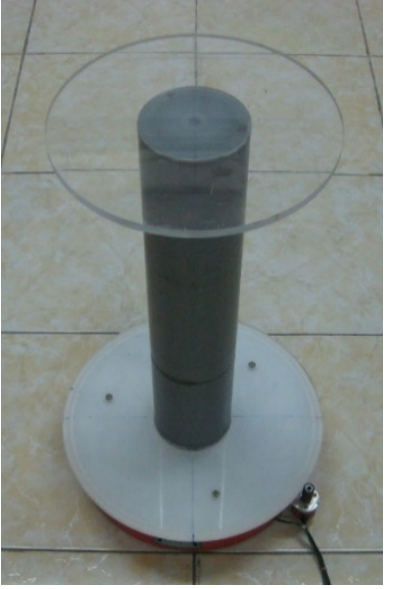

Figure 7. Rotor

- Tray: lifting the examined objects.

- Cylinder: linking swivel base and tray, supporting lifting vertically.

- Swivel base: rotating and lifting parts above.

- Rotation encoder: converting the angular position of the shaft to put into computer for checking the position of the rotating shaft.

- The data acquisition steps using rotor:

- Put radar toward the rotor in a certain distance.

- Adjust the swivel base to the original position.

- Turn on the radar and waiting for a short time for stabilization.

- Get the reference signal of surroundings.

- Place the object onto the tray.

- Control swivel base rotating to the desired angle.

- Collect measurable signal at each location and rotation angle.

- Process the collected signals.

\section{RADAR IMAGING Principles}

\subsection{A-scan and One-Dimensional image (1D)}

A-scan is considered transmitting a single pulse then receiving the echo.

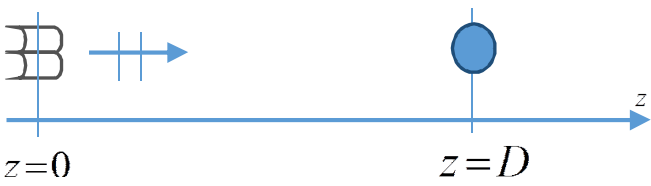

Figure 8. Outbreak source model

Using A-scan can restore one-dimensional image, assuming that at the position $z=D$ the scattered points with coefficient $\beta$, wave velocity ratio in the environment is $c, A$ is the wave amplitude, the reflected waves obtained in frequency domain format:

$$
S(\omega)=\rho A e^{-j 2 \frac{\omega}{c} D}
$$

Perform inverse Fourier transform to convert signals on the time domain we have:

$$
s(t)=\frac{\rho A}{\sqrt{2 \pi}} \delta\left(t-2 \frac{D}{c}\right)
$$

To mapped $t$ into one axis $z$ instead of $z=\frac{c t}{2}$ into formula (3) we have:

$$
s(t)=\frac{\rho A}{\sqrt{2 \pi}} \delta(z-D)
$$

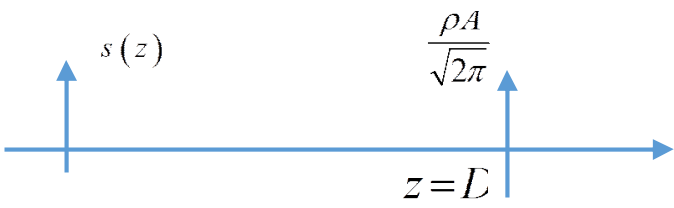

Figure 9. Distance to scattering point

\subsection{B-scan and Two-Dimensional image (2D)}

B-scan is actually just a set of A-scan in a given direction, for example in the $y$, separate each A-scan displacement amounts $\Delta y$. With this work, we obtained additional information reflected wave direction $y$. That is the basis of scattering restore $2 \mathrm{D}$ image. 


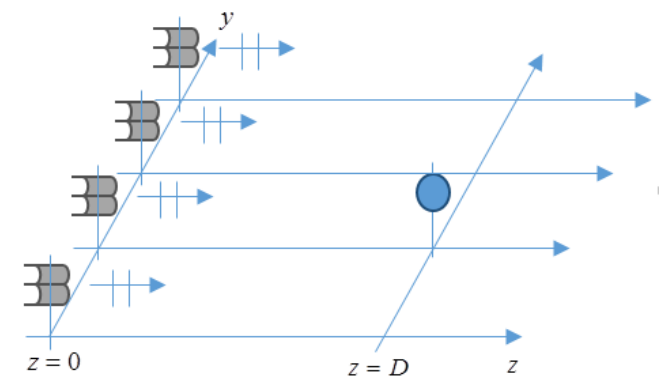

Figure 10. The set of A-scan

If now we apply the model as A-scan but with the data obtained is B-scan and display all the dirac pulse on 2-dimensional image obtained, it is a hyperbolic shape. Cause this image is shaped in terms of latency due to the wave propagation time on the unequal distances to the scattering point.

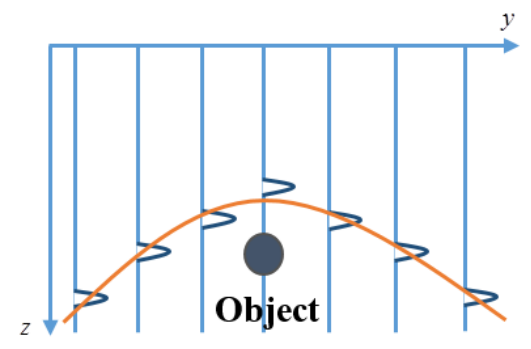

Figure 11. Photo reproduced by B-scan

\subsection{C-scan and Three-Dimensional image (3D)}

With the same principle as B-scan, if now we gathered all B-scan, the information we obtain an added dimension wave is the other dimension. Of course, C-scan will restore the object's 3-D image of the location in space, if object has large-sized theoretically, it will restore the shape of object's surface.

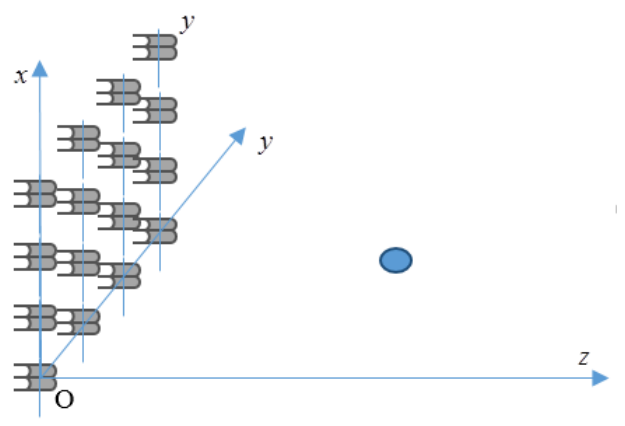

Figure 12. The set of B-scan

\subsection{Principle of Radar Operation}

Incident wave

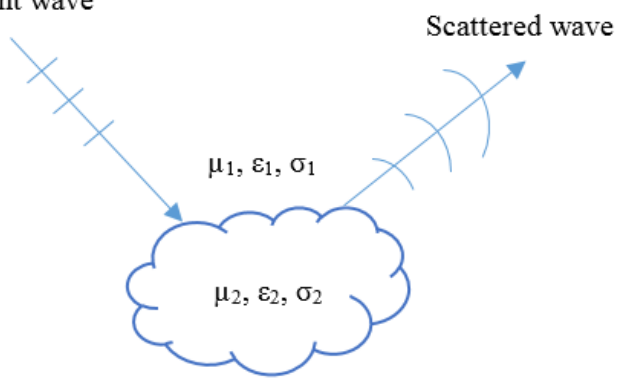

Figure 13. The principle of electromagnetic wave scattering

When the electromagnetic wave propagates in a determined environment $\left(\mu_{1}, \varepsilon_{1}, \sigma_{1}\right)$ and encounters a different environment $\left(\mu_{2}, \varepsilon_{2}, \sigma_{2}\right)$, it makes specific parameters of environment change suddenly and makes wave scatter in different directions, depending on the surface that wave impacts. After scattering, waves will be weaker in terms of amplitude, change in terms of propagating direction and phase. Based on these physical properties, researchers have created different generations of radar (active radar) following the under simple model:

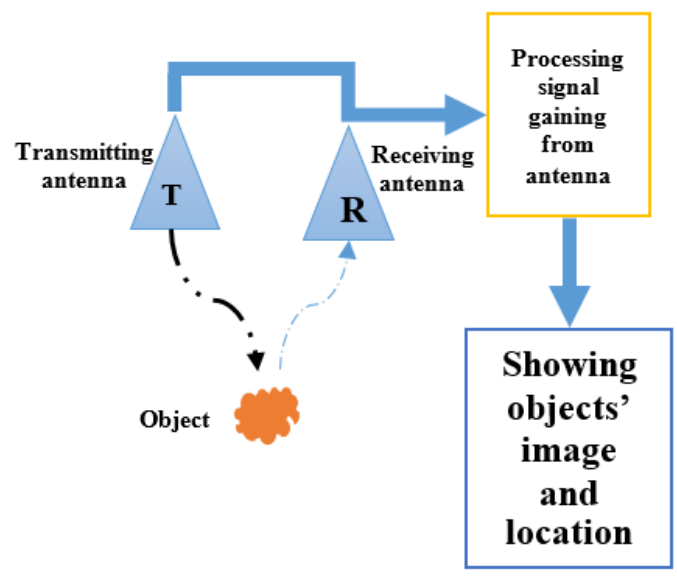

Figure 14. Radar diagram

\section{Development of DETECTING Hiden Objects Algorithm}

With the use of the rotor, this research mainly uses Hyperbolic Summation algorithm (HS). The 
title focuses on examining objects (metal sticks) when placed in the air and in the sand, thereby developing algorithm for these cases.

The examination was conducted with specimens which are metal sticks placed vertically in styrofoam box in the cases of with sand and without sand. Styrofoam is predetermined, known the size of the space inside the box.

\subsection{Hyperpolic Summation Algorithm}

Hyperbolic summation (HS) is the method of calculating the total scattering received from the object. Assume that, transceivers and scattering are points. The space that we observe will be divided into a set of points with $(\mathrm{x}, \mathrm{y}, \mathrm{z})$ coordinates, at the position of the scattering object, the total scattering will be greater and vice versa in location without object, collected value will be small.

If the signal received at $z=0$ is a discrete value of $\Psi\left(x_{i}, \mathrm{y}_{i}, z=0, t\right)$ where $i=1,2, \ldots I$, $j=1,2, \ldots J$ and $c_{m}$ is wave speed in environment, migration point matrix is calculated by the following formula:

$$
F_{H S}(x, y, z)=\sum_{i=1}^{I} \sum_{j=1}^{J} \Psi\left(x_{i}, y_{j}, z=0, t=\frac{2 R_{i, j}}{c_{m}},\right.
$$

If just calculating for $2 \mathrm{D}$ images:

$$
F_{H S}(y, z)=\sum_{j=1}^{J} \Psi\left(y_{j}, z=0, t=\frac{2 R_{j}}{c_{m}}\right)
$$

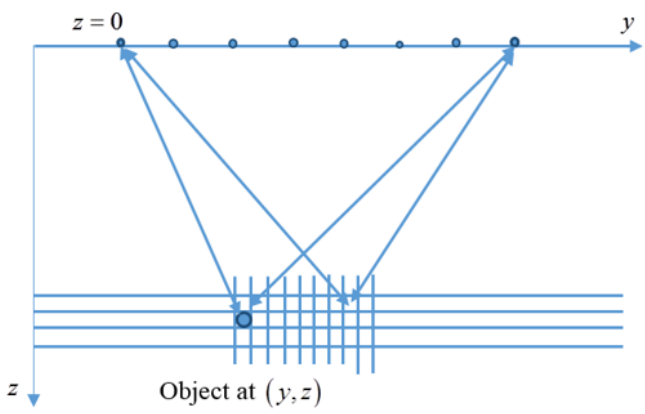

Figure 15. Coordinate system in HS algorithms
HS is the simplest method of reproduction techniques in radar object. This algorithm calculates on the time domain and it is easy to understand and is the basis for the algorithm developed later.

\subsection{Solution}

By determining the point between the beam away from the radar to the cell in the space of a box, we determine the distance from the radar to the cutoff point and from cutoff point to the examined cell. Thereby, we adjust the transmission time intervals in different environments in practical to help object detection accurately.

The steps are described in the following diagram and algorithm flowchart:

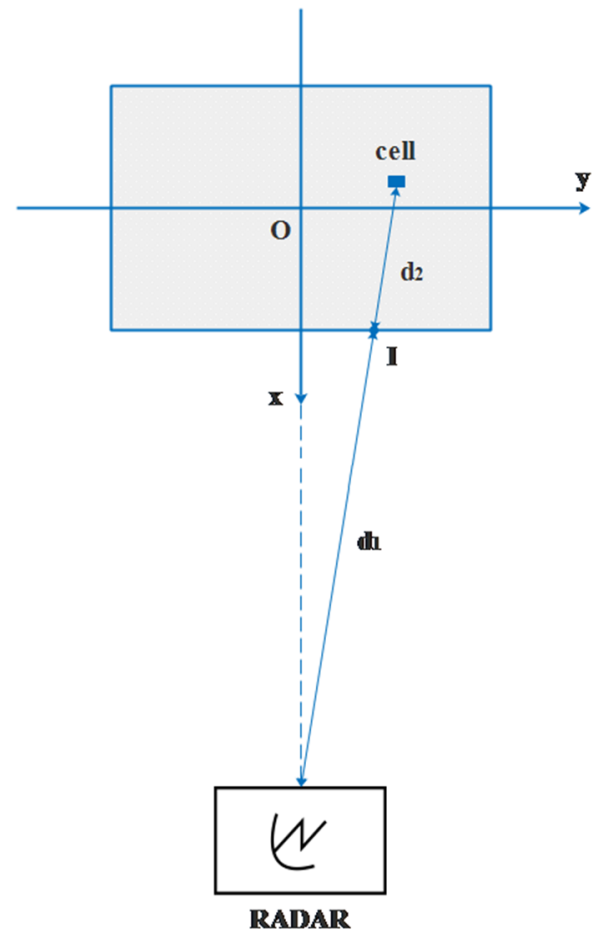

Figure 16. Space model 


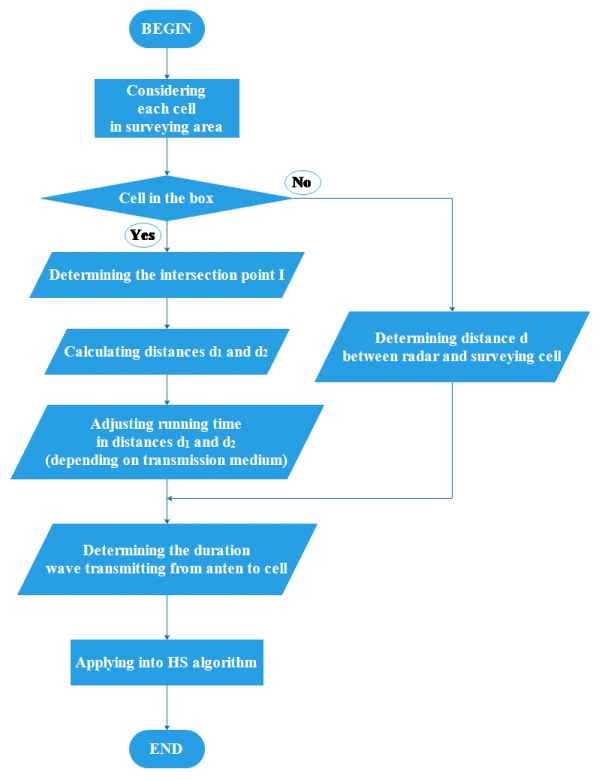

Figure 17. Algorithm diagram

\subsection{Testing on cases}

- Case 1: Two metal sticks

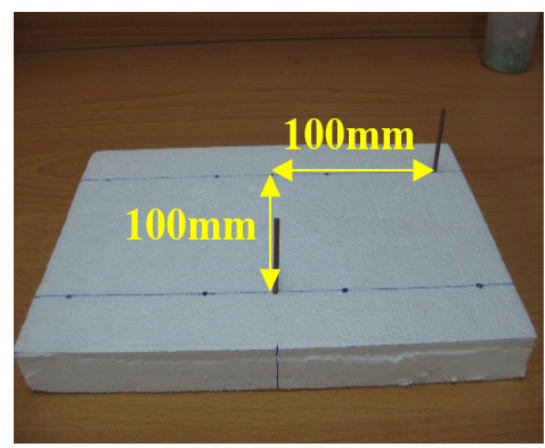

Figure 18. Two metal sticks lopsided

Styrofoam box without sand inside

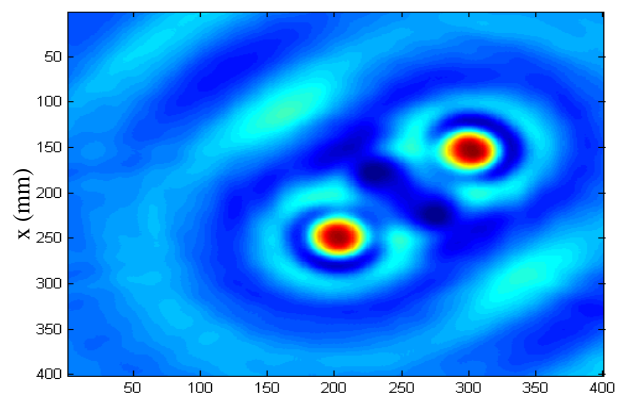

Figure 19. Applying HS algorithm for the case two metal sticks lopsided in styrofoam box without sand inside

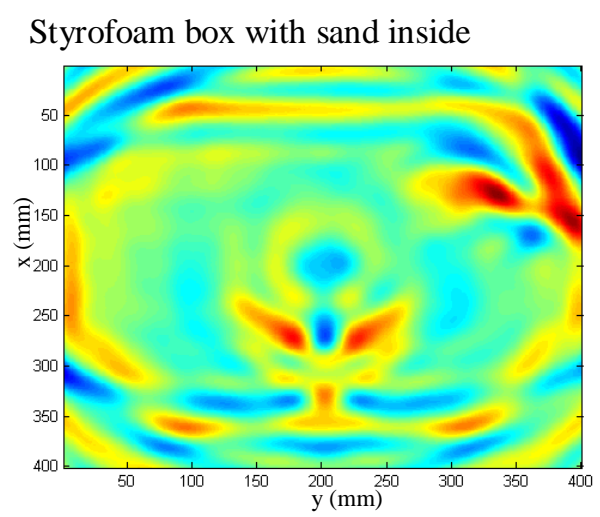

Figure 20. Applying HS algorithm for the case two metal sticks lopsided in styrofoam box with sand inside

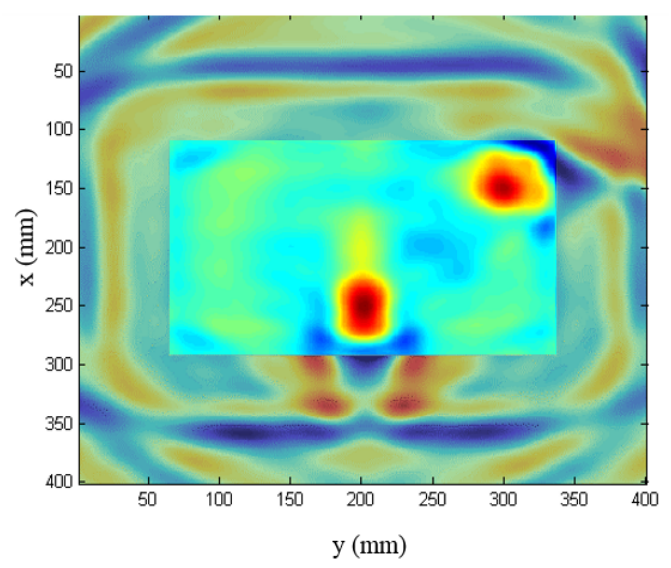

Figure 21. Applying improved HS algorithm for the case two metal sticks lopsided in styrofoam box with sand inside

- Case 2: Six metal sticks

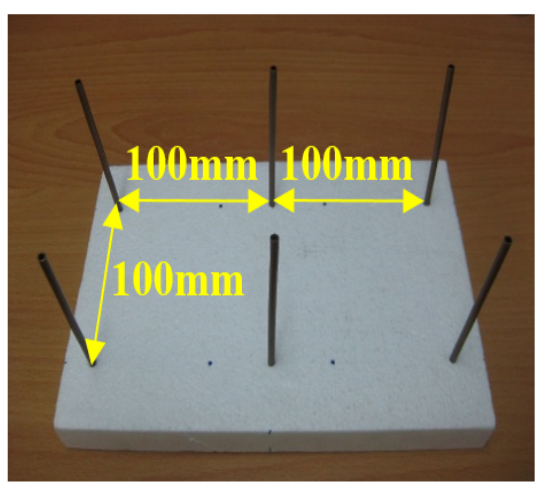

Figure 22. Six metal sticks 
Styrofoam box without sand inside

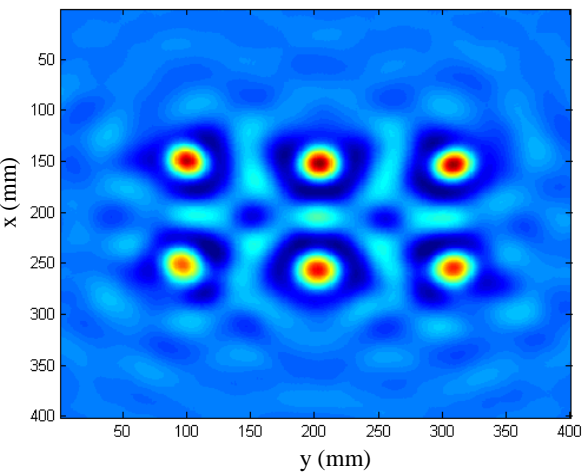

Figure 23. Applying HS algorithm for the case six metal sticks in styrofoam box without sand inside

Styrofoam box with sand inside

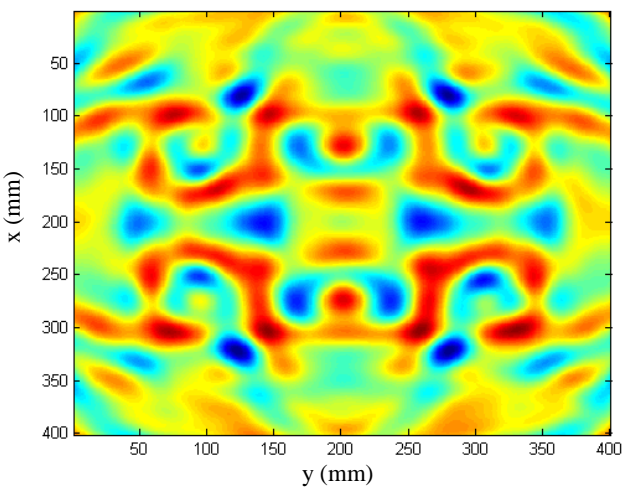

Figure 24. Applying HS algorithm for the case six metal sticks in styrofoam box with sand inside

\subsection{Evaluation}

- The solution has improved HS algorithm to detect the metal sticks in the sand that normal HS algorithms cannot.

- The time needed to process improved HS algorithm is around 2 minutes for 400x400 image (PC Core i5 2.6GHz, 4GB RAM).

- Objects identification in the image can be clearly distinguished and visible with relative accuracy.
- Metal stick identification image in the sand after processing can be identified like in the air case.

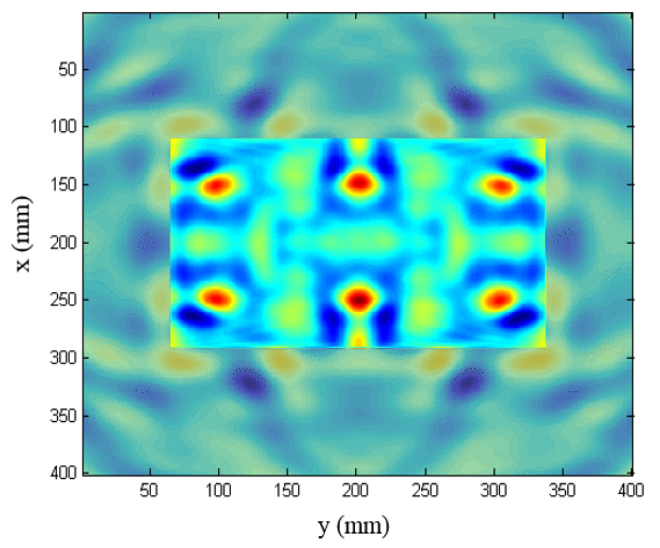

Figure 25. Applying improved HS algorithm for the case six metal sticks in styrofoam box with sand inside

\section{CONCLUSION}

The UWB radar system for detecting hidden objects has developed.

Improved HS algorithm helps detect metal objects in the space areas outside of air, thereby determining the relative position exactly in these space areas.

Algorithms can fully apply to the radar that does not need high precision, such as regular ground radar, hollow object scanners. It may apply for the examination of metallic objects hidden or located in special space with environments different from air. For example, examination of metal in reinforced concrete, metal objects lying in the sand... It can be improved more on algorithms and data processing techniques to apply in different physical environments.

\section{ACKNOWLEDGEMENT}

This research is funded by Vietnam National University Ho Chi Minh City (VNU-HCM) under grant number C2014-20-08. 


\section{Phát triển hệ thống phát hiện đối tượng ẩn dựa trên radar xung UWB}

- Trần Đại Thắng

- Đỗ Hồng Tuấn

- Hoàng Mạnh Hà

Trường Đại Học Bách Khoa, ĐHQG-HCM, Việt Nam

\section{TÓM TÁ́T}

Bài báo trình bày một giải pháp phát hiện đối tượng ẩn dựa trên radar xung UWB. Trong bài báo này, chúng tôi giới thiệu tổng quan về hệ thống UWB, những lý thuyết liên quan và việc sử dụng nó trong việc thực hiện đề tài. Các bước thu thập dũ liệu, xử lý dữ

Tùr khóa: Radar UWB, Giải thuật Migration, HS (Hyperbolic Summation).

\section{REFERENCES}

[1]. Augustin Jean Fresnel, "Plane Waves and Wave Propagation”, Nov 2011.

[2]. Julius Adams Stratton, "Electromagnetic Theory", Mcgraw Hill Book Company, 1941.

[3]. Karl Svozil, "Mathematical methods of theoretical physics", Edition Funzl, Oct 2013.

[4]. José M. Carcione, Laura Piñero Feliciangeli, and Michela Zamparo, "The explodingreflector concept for ground-penetrating-radar modeling", Annals of Geophysics, June 2002.

[5]. T. C. Bache, T. G. Barker, J. T. Cherry, N. Rimer, and J. M. Savino, "Explosion source liệu thông qua thuật toán phát hiện vật thể và việc phát triển thuật toán phát hiện đối tượng ẩn trong một số trường hợp sẽ được trình bày. Chúng tôi cũng đánh giá các kết quả đạt được, đưa ra các kết luận cho việc ứng dụng và hướng phát triển cho đề tài. 